\title{
CAOS \& COMPLEXIDADE: DESIGN DE SUPERFÍCIE E OS NOVOS PARADIGMAS DA CIÊNCIA
}

\author{
Mariana Laranjeira \\ PPGD - Faculdade de Arquitetura, Arte e Comunicação/UNESP \\ marilaranjeira.designer@gmail.com \\ João Fernando Marar \\ Faculdade de Ciências, Departamento de Computação/UNESP \\ fermarar@fc.unesp.br
}

\begin{abstract}
Resumo: As possibilidades expressivas dentro do campo do design de superfície vêm se apresentando cada vez maiores com o surgimento de tecnologias que permitem a construção de formas e estruturas de alta complexidade, como a impressão 3D. A fim de estabelecer uma relação entre design de superfície e os sistemas complexos, este trabalho busca abordar a significativa inter-relação dos novos paradigmas da ciência, concebidos a partir de conceitos como caos, complexidade e auto-organização juntamente com a cibernética e o design paramétrico, pressupondo assim, a consequente influência destes no processo de criação e construção de superfícies. Partindo da investigação da aplicabilidade das bases conceituais mencionadas, serão exemplificadas as perspectivas do design de superfície seja ele, arquitetônico, têxtil ou de qualquer outro caráter, produzido em uma primeira instância através de interfaces computadorizadas, designando-se a emergência de novos processos criativos e tecnologias. Além disso, elucidar a biomimética e sua importância na concepção do design, enquanto meio de inspiração nos sistemas complexos da natureza.
\end{abstract}

Palavras-chave: Complexidade, Design de Superfície, Biomimética, Impressão 3D.

Abstract: The expressive possibilities within the field of surface design come up with increasingly larger with the emergence of technologies that allow the construction of forms and structures of high complexity such as threedimensional printing. Establishing a relationship between design and complex systems, this work seeks to address the significant interrelationship of new paradigms of science, designed from concepts such as chaos, complexity and selforganization along with the cyber and parametric design, assuming thus the consequent impact of these in the creation and construction of process surfaces. Starting from the investigation of the applicability of the aforementioned conceptual bases, will be exemplified prospects of surface design it is architectural, textile or any other character, produced in the first instance 
through computer interfaces, assigning the emergence of new creative processes and technology. Furthermore, elucidating biomimetics and its importance in the design of the design as a means of inspiration in complex systems of nature.

Keywords: Complexity, Surface Design, Biomimetics, 3D Printing.

\section{INTRODUÇÃO}

Destacando principalmente a física e a matemática, as ciências passaram por uma significativa evolução a partir de teorias para além do determinismo, como a Teoria do Caos e a consequente abordagem dos Sistemas Complexos Dinâmicos e da Auto-Organização, chegando inclusive a revisar seus princípios e teorias. Atingindo, entretanto, muitas áreas de conhecimento, como menciona Prigogine (2002), da cosmologia à economia, a consciência de caos e complexidade permite a emergência de novos paradigmas importantes para o iminente desenvolvimento de pesquisas no campo do design da forma.

Tenu (2009) determina a auto-organização como conceito diretamente associado com a complexidade e frequentemente encontrado no ambiente natural. Apesar disso, é possível conceber uma transdisciplinaridade com outros campos de conhecimento como a engenharia de materiais e o design, implicando por consequência, na construção de superfícies complexas. Como complementação, Theodorou (2012) menciona que se absorvermos a essência da característica de autoorganização, presente principalmente em processos de morfogênese na natureza, então será possível a criação de uma enorme variedade de formas.

Investigando esses novos paradigmas da ciência, a pesquisa gera oportunidade para a discriminação das inter-relações ocorridas com a cibernética e algoritmos desenvolvidos em meio computacional, ou no referido design paramétrico que se apoia em parâmetros desenvolvidos digitalmente, para geração de formas e suas consequentes superfícies. Para a criação de superfícies complexas, podemos considerar o que afirma CHANDRASEGARAN et.al. (2013) de que o desenvolvimento de produtos na contemporaneidade exige modelagem e simulação de muitos aspectos do produto em meio digital, como a aparência e a forma, para que apenas então seja realizada a construção real do objeto.

Em uma segunda instância, é significativo mencionar a tendência e o crescimento da biomimética e da biônica, que de maneiras similares permitem, não apenas no campo do design, como em outras áreas da ciência, a observação da natureza e de seus sistemas complexos como inspiração para a projeção de novas superfícies que apresentem intensa complexidade de forma e estrutura. Oxman (2011) destaca o alto nível de integração e precisão das estruturas naturais e a importância da absorção destas características para a construção de superfícies. Superfícies estas, permitidas atualmente pelo advento de tecnologias como a impressão 3D.

\section{DESENVOLVIMENTO}

Pode parecer coerente para muitos que, na contemporaneidade, vivemos em um mundo complexo, absorto em relações de complexidade entre todos os seus elementos, sejam eles as pessoas, as sociedades, e até na natureza que nos rodeia. 
Mas a percepção da complexidade, nem sempre significa o seu entendimento, e muito menos a deliberação de se adentrar nos caminhos insólitos de sua investigação. Heylighen (1988) observa que entender a complexidade parece ser a única possibilidade para assimilar essa evolução na qual tudo parece estar se tornando cada vez mais incerto e mutável.

Dentro do que conhecemos como história da humanidade e da ciência, o ser humano vem, ao longo dos tempos, se enredando por pensamentos determinísticos. 0 determinismo se caracteriza principalmente pela causalidade. Ele designa que um evento tem seu comportamento determinado pelas suas condições iniciais, sem elementos aleatórios envolvidos. A ciência tradicional, em campos como os da física, química e matemática, se fundamenta no determinismo.

Entretanto, principalmente no fim do século XIX e início do século XX, a ciência passa por uma evolução ao surgirem questionamentos sobre as teorias puramente determinísticas e com a consequente geração de outro viés do conhecimento e de uma nova forma de racionalizar. É neste novo segmento aonde irá se embasar esta pesquisa, na concepção da complexidade.

Citando Montaner, Ramos (2002) afirma que a tendência da contemporaneidade é reagir ao paradigma da simplificação que vem dominando nossa cultura,

\begin{abstract}
"O paradigma da complexidade virá do conjunto de novas concepções, novas visões, novas descobertas e novas reflexões que vão se conciliar e reunir. (...) o pensamento simplificante se funda sobre o domínio de dois tipos de operações lógicas: disjunção e redução, que são uma e outra brutalizantes e mutilantes, então os princípios do pensamento complexo serão necessariamente princípios de distinção, de conjunção e de implicação." (RAMOS, 2006 apud MONTANER, 2002).
\end{abstract}

Inicialmente vinculados à ciência, os novos paradigmas tem como base conceitual as teorias do caos e da complexidade, juntamente com suas implicações referente à auto-organização e ao pensamento sistêmico. Permitindo uma assimilação mais realística da estrutura dos sistemas.

A teoria do caos aborda os sistemas complexos que apresentam característica de não linearidade e instabilidade, com grande número de interações entre seus componentes. Ela surge como consequência de uma série de investigações ocorridas ao longo do desenvolvimento da ciência por nomes como Henri Poincaré e Edward Lorenz. No final do século XIX, Poincaré propõe um estudo da mecânica de corpos celestes, conhecido como Problema dos Três Corpos e contribui assim para a pesquisa de sistemas dinâmicos complexos ao identificar a presença de caos na interação celestial. Lorenz, por sua vez, respaldado por máquinas de cálculo, propõe o que ficou conhecido como Efeito Borboleta, através da concepção de um atrator estranho que mapeia a evolução dos sistemas dinâmicos caóticos, estabelecido em um padrão complexo. Esta teoria do Efeito Borboleta questiona o fato de uma mínima alteração de informação em um sistema gerar resultados absurdamente contrários daqueles propostos inicialmente.

Referindo-se a Lorenz e a sua contribuição para a teoria do caos, Chapman (2009) explica que quando os pequenos computadores surgiram, permitiram a execução de simulações de sistemas complexos, possibilitando aos teóricos a compreensão de que existe uma classe de sistemas que nunca entram em equilíbrio, e 
nunca repete um estado anterior. Chapman (2009) também aponta, de maneira resumida, que as características presentes no comportamento caótico podem ser de que o sistema é determinístico, apresenta um atrator estranho e nunca repete a si mesmo. Complementa ainda que, ao contrário do que inicialmente a teoria do caos propõe, o sistema não precisa ser aleatório, ele pode simplesmente ser um sistema determinístico que é computationally irreducible.

A aspiração do conceito de caos propicia na configuração da base para a teoria da complexidade. A complexidade lida com os sistemas complexos juntamente com seus elementos e as interações entre eles. Heylighen (1988) descreve a complexidade e a compara com o conceito matemático de rede (network), constituída de nós (nodes) com conexões emergindo entre eles.

Quando nos referimos aos conceitos de caos e complexidade, é interessante mencionar também que apesar da similaridade, os dois não são a mesma coisa. Filho (2008) apresenta essa diferença ao citar Baranger que por sua vez afirma que,

\footnotetext{
“O gás simples é caótico, mas não é complexo no sentido atual. Complexidade e caos têm em comum a propriedade da não linearidade todo sistema não linear é caótico em alguma parte do tempo o que significa que complexidade implica a presença do caos, mas o inverso não é verdadeiro." (FILHO, 2008)
}

Caracterizando-os pelo requisito de não linearidade, Chapman (2009) reflete sobre sistemas caóticos e complexos, afirmando que as várias interações apresentam propriedades de emergência.

\footnotetext{
“Essas propriedades são emergentes porque não podem ser previstas com base no que se sabe sobre as partes ou suas relações. Até agora, tais sistemas podem ser modelados em computadores, para que possa ser encontrado o que realmente emerge de um conhecido grupo de interações, mesmo que não as possamos prever." (CHAPMAN, 2009)
}

A partir disso, podemos afirmar que os sistemas complexos podem ou não apresentar regras completamente definidas a priori, mas devido à grande quantidade de interações, a imprevisibilidade será uma regra claramente observada em algum nível ou instância deste sistema e a sua organização será emergente. Oliveira (2001) observa que essa imprevisibilidade irá gerar padrões de comportamento, ou seja, ordem que nasce do caos gerando sistemas complexos através da auto-organização espontânea em sistemas físicos e químicos simples.

Considerando os primórdios dos estudos de auto-organização é importante absorver 0 trabalho de Alan Turing sobre a morfogênese, processo de desenvolvimento e crescimento dos organismos. Turing (1953) observa que na fase inicial da morfogênese, há a presença de simetria, homogeneidade e equilíbrio, características que bloqueariam o crescimento e se manteriam para sempre se fossem levadas em consideração as leis elementares de eletricidade, magnetismo ou de mecânica quântica. No entanto, rompendo com o previsível, isso não ocorre, pois há a presença de perturbações aleatórias que geram instabilidade no sistema e permitem o seu crescimento, até que este atinja novamente um estado de equilíbrio. 
A partir dessas constatações, Turing desenvolveu equações não-lineares demonstrando que a reunião de elementos simples, seguindo regras simples, poderia formar estruturas e organismos complexos.

Complementando essa teoria, Filho (2008) cita Capra que determina que,

\begin{abstract}
“A auto-organização é a emergência espontânea de novas estruturas e de novas formas de comportamento em sistemas abertos, afastados do equilíbrio, caracterizados por laços de realimentação internos e descritos matematicamente por meio de equações não lineares". (FILHO, 2008)
\end{abstract}

Tais equações não lineares serão então, com a ocorrência da cibernética, transformadas em algoritmo e utilizadas para aplicação do conceito da complexidade em inúmeros campos de conhecimento. Afinal, como nos mostra Chapman (2009), "a complexidade é a nova unificadora das disciplinas (interdisciplinaridade), inclusive fazendo a ponte entre ciência e sociedade." (CHAPMAN, 2009)

\title{
2.1 Tecnologias emergentes
}

\subsubsection{Cibernética}

Quando evoluímos um pouco na história da ciência, adentramos em um ramo que em sincronia com os novos paradigmas de pensamento, mencionados anteriormente, influenciou em uma nova era de informação, a cibernética.

A cibernética traz à tona as relações do homem com as máquinas, em um estudo de comunicação e gerenciamento de informações inserido dentro do contexto da teoria dos sistemas. Ashby (1970) afirma que a cibernética,

\begin{abstract}
“(...) oferece um vocabulário singular e um conjunto singular de conceitos adequados à representação dos mais diversos tipos de sistemas (...) a cibernética deverá, provavelmente, revelar grande número de paralelismos interessantes e sugestivos entre a máquina, o cérebro e a sociedade. E pode prover uma linguagem comum através da qual, descobertas em um ramo possam ser prontamente utilizadas em outros." (ASHBY, 1970)
\end{abstract}

Muito além desta definição, podemos tratar a cibernética como conceito de fundamental importância nos princípios da complexidade. Ashby (1970) introduz o estudo da cibernética afirmando que a mesma esta entre os métodos capazes de gerar um tratamento científico do sistema para lidar com a complexidade e, que a complexidade se apresenta constantemente no mundo biológico.

Dessas considerações, podemos contemplar que, assim como a complexidade, a cibernética também é um campo transdisciplinar, o que nos leva a perceber que o pensamento sistêmico é realmente uma tendência na ciência contemporânea, abrindo portas para a construção do conhecimento e o crescimento das tecnologias. Como um caminho para lidar com a complexidade, Heylighen (2007) considera a cibernética dotada de conhecimento subjetivo e inserida dentro dos preceitos da complexidade, afirmando que ela,

"Junto com as teorias de auto-organização e biologia evolutiva, nos mostra que regularidade e organização não são fornecidas, mas que a emergência dinamicamente surge como um conjunto de forças conflitivas e flutuações 
randômicas, um processo habilmente denominado como 'order out of chaos'". (HEYLIGHEN, 2007)

Comparada assim com a teoria da informação, a cibernética também abre caminhos para lidar com um conjunto de possibilidades e permitir o advento de técnicas para processamento de dados. Desta forma, inserida dentro da cibernética, a ciência da computação, juntamente com o desenvolvimento da robótica, irá desencadear o surgimento de meios de expressão como o CAD (computer aideddesign), utilizado fortemente no design de produto.

Com o CAD, o desenvolvimento matemático de formas em meio computacional pode ser visualizado digitalmente com precisão antes de o produto ser efetivamente produzido. Menges (2012) explica a importância de se antecipar no processo de construção do objeto,

\footnotetext{
"A lógica de sistemas geradores computacionais que integram material, forma e desempenho nos processos de design oferecem maneiras de processar o fluxo de forças estruturais e de interação com as influências ambientais sobre um material de construção e equilibrar diferenciação morfológica com as características e o comportamento dos materiais." (MENGES, 2012)
}

Essa antecipação, principalmente quando nos referimos à constituição de objetos complexos, é essencial, permitindo economia de tempo e dinheiro. Deste modo, as possibilidades expressivas passam a aumentar gradativamente e é possível conceber superfícies altamente complexas virtualmente.

Com a formação virtual do objeto, a execução do mesmo fica a cabo de processos e máquinas que sejam capazes de reproduzir fielmente os elementos propostos, independente de sua complexidade. Um dos processos que mais corresponde a estas expectativas atualmente é a técnica de impressão 3D.

\subsubsection{Impressão 3D}

A tecnologia da impressão 3D surge como um processo que vem ganhando grande repercussão por permitir uma aceleração nas técnicas de prototipagem. Inicialmente proposta para o campo da engenharia, a impressão 3D se apresenta inserida nos conceitos da cibernética e parece, cada vez mais, acometer muitas áreas da ciência, se tornando uma ferramenta hábil para facilitar tanto no trabalho de pesquisadores como de empresas e indústrias. Aderida no design de produtos, a sua disseminação atinge também muitas áreas como as artes, cinema, arquitetura, passando pela medicina e inclusive a nanotecnologia. Com isso, não é difícil afirmar sobre sua importância na sociedade pós-moderna e na influência que emite nos processos de criação e fabricação, inserida automaticamente dentro do contexto do design. E, no caso deste trabalho, mais especificamente no design de superfícies.

A sua principal característica está na constituição de formas e estruturas a partir de parâmetros propostos em meio computacional. Estes, com base em cálculos matemáticos e porque não, em algoritmos gerados a partir de equações não-lineares, fornecem informação para o maquinário gerar o protótipo através do depósito acumulativo de camadas de um determinado material, ou uma mescla de materiais variados. Com isso, há também o desencadeamento de pesquisas com relação aos materiais utilizados, que ainda que sejam variados, podem ser cada vez de mais tipos. 
Mas suas vantagens não se apresentam apenas no processo, elas estão também nos resultados obtidos: objetos que dificilmente seriam reproduzíveis de outra maneira. A partir de então, não apenas fica possível a concepção de superfícies altamente complexas, como também na sua construção e reprodução por meio de novos materiais. Com isso, as limitações criativas passam a depender principalmente do designer e não apenas dos tecnológicos disponíveis.

\subsubsection{Biomimética}

Podemos considerar as informações levantadas neste trabalho sobre as observações de Alan Turing, assim como de muitos outros pesquisadores, sobre a presença assídua de sistemas complexos na natureza.

Com bilhões de anos de existência, o planeta Terra passou por mudanças e distúrbios até atingir o que, ainda pouco, conhecemos dele. Toda essa evolução permitiu que suas superfícies, formas e estruturas alcançassem níveis de equilíbrio e perfeição como nenhum processo artificial poderia. Oxman (2011), ao mencionar Benyus, sustenta que,

\footnotetext{
"Estruturas naturais possuem um alto nível de integração e precisão com suas funções. Um traço distintivo fundamental do design da natureza é a sua capacidade no mundo biológico para gerar estruturas complexas de orgânicos ou inorgânicos." (OXMAN, 2011, apud Benyus, 1997).
}

Nada como a natureza para nos fornecer belos e magníficos exemplos de complexidade inigualável, superfícies naturais oferecem exemplos de notáveis propriedades diversas. (EADIE \& GHOST, 2011) Desta maneira, é praticamente impossível que, na contemporaneidade, aquele que se proponha a conceber formas e estruturas complexas, seja o engenheiro, o arquiteto ou mesmo o designer, não se inspire ou absorva as características de algum recurso do meio natural. Oxman (2011) fundamenta sua investigação não apenas nas formas da natureza como também nas características de sua constituição, a fim de se concentrar conhecimento para a constituição de novas tecnologias. As propriedades que determinadas superfícies apresentam, estão diretamente relacionadas com sua formação estrutural e sua composição material. Oxman (2011) afirma que a função destes materiais naturais explora suas propriedades estruturais excepcionais e que essas relações tem implicações significativas para o projeto de engenharia de materiais mecanicamente eficientes.

A Biomimética é uma das ciências que permite a incorporação de um estudo da natureza para a geração de materiais e processos. Koch et. al. (2009) consideram que as superfícies biológicas e sua diversidade estrutural, fornecem propriedades multifuncionais para a produção de materiais funcionais biomiméticos.

Além disso, a Biomimética, como campo transdisciplinar, se tornou altamente considerada no processo criativo, como nos revelam Eadie e Ghost (2011),

\footnotetext{
"Biomimética, em sua interpretação mais estrita, é o processo de emulação de formas da natureza, de encontrar uma solução, incluindo o 'design' e o 'fazer' com o menor impacto ambiental. Na verdade, os sistemas biológicos devem ser vistos mais como geradores de conceito, em termos de transferência de princípios e mecanismos ao invés de algo literalmente para
} 
copiar. As tecnologias modernas tornaram possível projetar e fabricar produtos/sistemas que são baseados na natureza." (EADIE \& GHOST, 2011)

Com o desenvolvimento da ciência no campo da complexidade e da exploração da tecnologia que a mesma acarreta, vimos que cada vez mais surge um cenário de crescimento de possibilidades criativas. A biomimética é com certeza uma das bases conceituais para essa evolução que abarca muitas áreas de conhecimento. Chen et. al. (2012) determinam que o projeto bioinspirado exigirá no futuro equipes multidisciplinares de designers e cientistas (bioquímica, biologia, fisiologia, biologia molecular, anatomia) para desenvolver materiais com estruturas complexas.

Assim, fica evidente que o progresso que atingem os materiais e as estruturas inspirados na natureza envolve novos desafios tecnológicos, aonde técnicas como a impressão 3D, apresentada anteriormente, são de fundamental importância.

\subsection{Design de superfície}

Quando falamos de superfície, não há como se desassociar dos estímulos sensoriais que esta produz e da importância destes na construção e determinação do objeto. Objeto que depende diretamente do vínculo da sua superfície com a sua forma e/ou volume, estabelecendo uma estrutura determinada. Allgayer (2009) define de uma maneira bem completa a superfície e suas propriedades,

\footnotetext{
"Superfícies rígidas ou flexíveis, cumprindo ou não a função de estruturação, podem assumir diferentes formas, sejam elas planas, curvas, contínuas ou fragmentadas. Independentemente de uma caracterização universal do seu propósito, é mister consensualizar seu papel como limite físico de volume. Caracterizadas como envolventes, podem bloquear ou irradiar calor, restringir ou franquear visuais, transitando entre diferentes graus de permeabilidade ou opacidade, proteção ou vulnerabilidade." (ALLGAYER, 2009, p.7)
}

Apesar de estar inicialmente associado ao design têxtil, o termo Design de Superfície pode ser assimilado para superfícies em geral como afirma Rubim (2010) de que independente de sua natureza, a superfície pode ser elemento principal de um projeto de design. Ainda no que diz respeito à definição desta área podemos considerar que o design de superfícies se encarregará de estudar diferentes tipos de superfícies, podendo elas ser bidimensionais ou tridimensionais, reais ou virtuais, e de constituição material variada. Em uma abordagem mais profunda, podemos concordar com o que define Ruthschilling (2008):

\footnotetext{
"Design de superfície é uma atividade criativa e técnica que se ocupa com a criação e desenvolvimento de qualidades estéticas, funcionais e estruturais, projetadas especificamente para constituição e/ou tratamentos de superfícies, adequadas ao contexto sociocultural e às diferentes necessidades e processos produtivos." (RUTHSCHILLING, 2008)
}

Allgayer (2009) define a superfície afirmando que "A forma, entendida como matriz sobre a qual os volumes se configuram, pressupõe a materialização de limites físicos vinculados à sua constituição espacial" (ALLGAYER, 2009)

Por ser um campo muito específico, referente à composição formal do objeto e à sua constituição material associada com as técnicas empregadas, o design de 
superfície é uma área interdisciplinar, estando muitas vezes associada com outras vertentes do design, com a engenharia de materiais ou com a arquitetura.

Além disso, o design de superfície é uma área com várias ramificações. Cada vertente está associada principalmente de acordo com os materiais e os processos utilizados no desenvolvimento do projeto, como por exemplo, os artigos de cerâmica, papelaria e têxteis.

O design de superfície contemporâneo toma uma interessante direção com o advento de tecnologias que permitem uma evolução nos processos de sua criação e construção. KICINGER et.al. (2005) confirmam que o design evolucionário deve integrar ideias de ciência da computação, engenharia e biologia evolutiva. A exploração de novas superfícies, e consequentes, formas e texturas, antes inimagináveis, surgem a partir de um paralelismo traçado entre estas áreas e a absorção dos princípios da teoria da complexidade.

\subsection{O Estado da Arte}

Através de vários métodos de concepção, alguns dos quais fazendo uso do processo de impressão 3D, o design de superfícies tem levantado inúmeras vertentes de pesquisa nas diversas áreas do design. A seguir, são apresentadas algumas pesquisas que tem como foco o conceito da complexidade.

Vlad Tenu é um arquiteto romeno que desenvolve projetos de superfícies para arquitetura. Seu foco é no projeto de superfícies mínimas, concebidas por algoritmos matemáticos. Inseridas no princípio da complexidade, suas estruturas de materiais diversos emergem no espaço através da auto-organização de componentes simples.
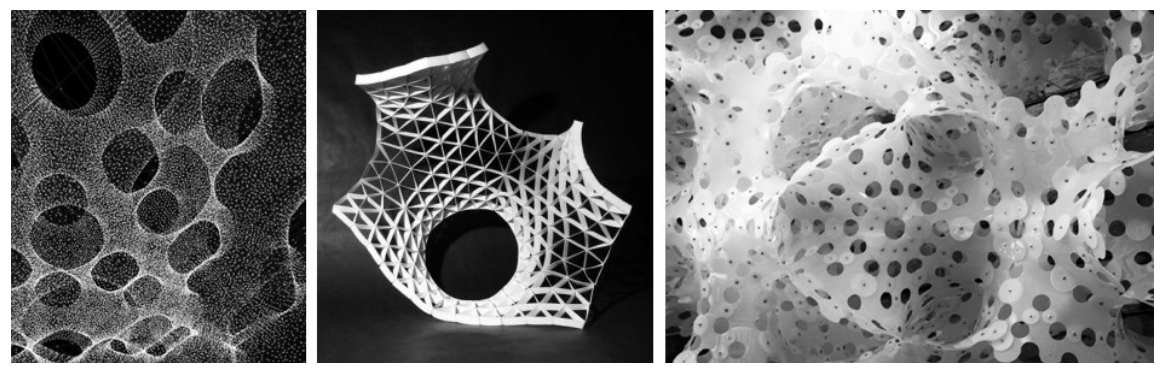

Figura 1 - Projetos de Vlad Tenu.

O Matsys Studio localizado na Califórnia, nos Estados Unidos, explora as relações entre design, arquitetura, engenharia, biologia e computação. Suas estruturas complexas emergem da organização de seus elementos. Também não utiliza a impressão 3D, mas faz uso de vários tipos de materiais como o papelão e a madeira.
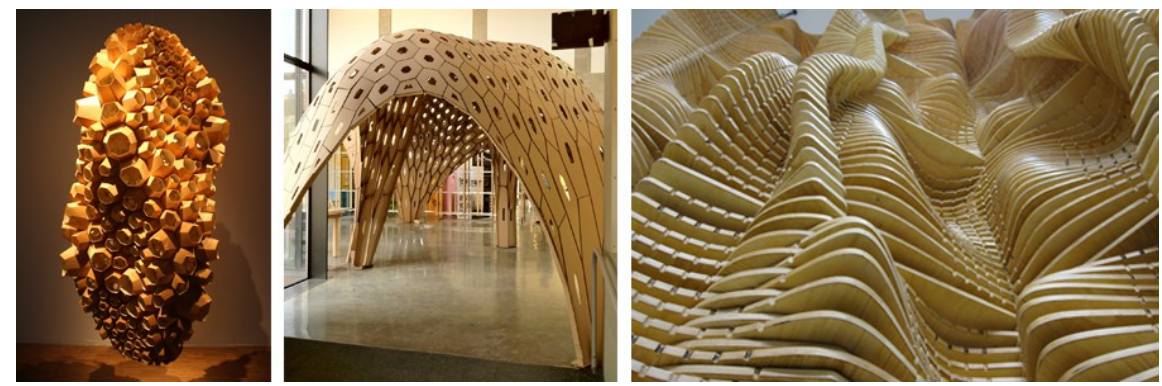

Figura 2 - Projetos do estúdio Matsys. 
Michael Hansmeyer é um arquiteto da Universidade de Columbia que executa propostas baseadas na impressão 3D de superfícies complexas. Projetos que, devido ao alto nível de complexidade, seriam impossíveis de se reproduzir sem essa tecnologia.
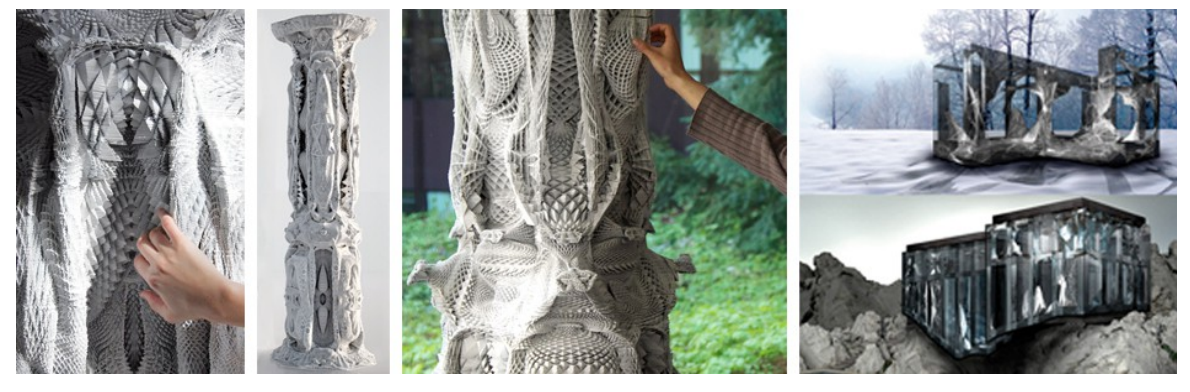

Figura 3 - Projeto de colunas impressas em 3D.

Neri Oxman é pesquisadora do Media Arts and Sciences no MIT (Massachusetts Institute of Technology). Ela foi tida como um exemplo pertinente a este trabalho por dois motivos. Em primeiro lugar, seus projetos se encaixam nas três bases fundamentais tratadas: a acepção da complexidade, o design bioinspirado e a exploração da tecnologia 3D. Em uma segunda instância, porque Oxman faz parte do MIT, instituto onde muitas outras pesquisas sobre os conceitos determinados, podem ser encontradas. Um exemplo de seu projeto é a luva Carpal Skin (Figura 4), que inspirada em animais, auxilia no tratamento de pacientes com Síndrome de Túnel do Carpo. As nervuras complexas da superfície têm a capacidade de aliviar a dor através das propriedades de sua estrutura e composição material. Outro projeto inspirado na complexidade da natureza é o Pregnancy Corset (Figura 5). O espartilho feminino foi confeccionado para grávidas em impressão 3D. As suas características de adaptação ao corpo da mulher emergem de acordo com a evolução da gravidez.

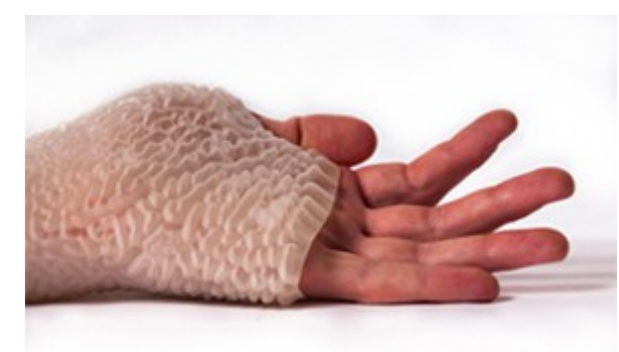

Figura 4 - Projeto Carpal Skin
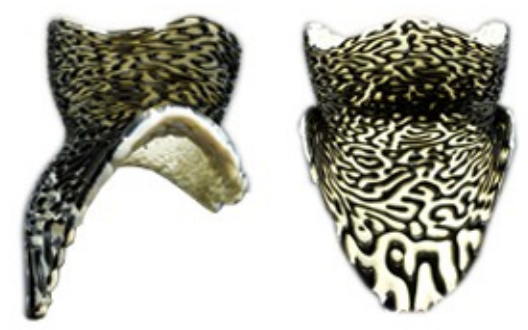

Figura 5 - Projeto Pregnancy Corset

Evidente que, além destes exemplos apresentados, existem muitos outros projetos que estão sendo desenvolvidos com os conceitos abordados neste trabalho. As pesquisas, em geral, ainda são primárias e conservam seu foco em novas superfícies, formas e estruturas que apresentem propriedades significativas para determinadas aplicações. No entanto, a partir destas pesquisas é que se dará margem para a criação de projetos completos, inseridos em muitas áreas de conhecimento. Além disso, percebe-se que a maioria das investigações ocorre no campo da engenharia e da arquitetura, e em centros de pesquisa no exterior. Buscando 
desenvolver a pesquisa no Brasil, é preciso que se crie um incentivo para a exploração dos atributos do design e a consequente evolução do ramo do Design de Superfícies.

\section{CONCLUSÃO}

A impressão 3D vem ganhando cada vez mais campo no mundo contemporâneo. As facilidades que esta tecnologia proporciona no processo de produção leva a um incentivo nas pesquisas que aumentem sua abrangência e provoque uma evolução de áreas como a engenharia, a arquitetura e o design. Desta maneira, o conteúdo bibliográfico pertinente às suas investigações é cada vez mais extenso nos diversos campos da ciência. Considerando os novos recursos tecnológicos necessários para a construção de superfícies e formas complexas, este trabalho buscou relacionar os conteúdos, a fim de apresentar a base epistemológica da concepção da complexidade, apontando as origens desta linha de pensamento e a sua importância no futuro.

Através de uma análise bibliográfica da teoria do caos, juntamente com a acepção da complexidade e da auto-organização, passando por áreas como a cibernética e o uso de máquinas, foi elucidado como a utilização de novas tecnologias associadas à criação de materiais e processos tende a tornar gradativamente o design como uma potente ferramenta transdisciplinar.

Deste modo, é significativo considerar que uma exploração das técnicas criativas que levem a novos caminhos da expressão artística, se beneficiando da inspiração nos sistemas complexos da natureza a partir da biomimética, possa propiciar o surgimento de projetos que apresentem significativa importância multidisciplinar com áreas como a medicina, por exemplo. Incidindo assim em projetos de design altamente eficientes.

Por meio da assimilação dos recentes paradigmas para o design contemporâneo, este trabalho determina a importância de uma atitude transdisciplinar com relação às informações, e mais especificamente, da associação do design de superfície com a biomimética e as novas tecnologias, além de inquirir o questionamento das reais contribuições e aplicações dos conteúdos tratados.

\section{REFERÊNCIAS}

ALLGAYER, Rodrigo. Formas Naturais e Estruturação de superfícies mínimas em arquitetura. 2009. Dissertação (Mestrado em Design e Tecnologia) - Universidade Federal do Rio Grande do Sul.

ASHBY, William Ross. Uma Introdução à Cibernética. São Paulo: Editora Perspecctiva, 1970.

CHANDRASEGARAN, Senthil Kumaran et.al. The evolution, challenges, and future of knowledge representation in product design systems. Computer-Aided Design, v. 45, p. 204-228, 2013.

CHAPMAN, Graham. Chaos and Complexity. International Encyclopedia of Human Geography, 2009.

CHEN, Po-Yu; MCKITTRICK, Joanna; MEYERS, Marc André. Biological materials: Functional adaptations and bioinspired designs. Journal Progress in Materials Science. Volume 57, Issue 8 de novembro, Pages 1492-1704, 2012. 
EADIE, Leslie \& GHOSH, Tushar K. Biomimicry in textiles: past, present and potential. An overview. Journal of The Royal Society. Interface 2011, v. 8, p. 761-775, 2011.

FILHO, Ladislau Batista de Oliveira. Complexidade em Processos Coorporativos: Caos, Auto-organização, Emergência e vieses Sistêmicos. Dissertação (Mestrado em Administração de Empresas) - Instituto Superior de Ciências do Trabalho e da Empresa, Lisboa. 2008

HEYLIGHEN, Francis. Building a Science of Complexity. 1988 Annual Conference of the Cybernetics Society - Cybernetics Society, King's College, London. 1988.

HEYLIGHEN, Francis; CILLIERS, Paul; GERSHENSON, Carlos. Complexity and Philosophy. J. Bogg and R. Geyer (editors) - Complexity, Science and Society, Radcliffe, Oxford, 2007.

KICINGER, Rafal; ARCISZEWSKI, Tomasz; JONG, Kenneth De Jong. Evolutionary computation and structural design: A survey of the state-of-the-art. Computers and Structures, v. 83, p.1943-1978, 2005.

$\mathrm{KOCH}$, Kerstin; BHUSHAN, Bharat; BARTHLOTT, Wilhelm. Multifunctional surface structures of plants: An inspiration for biomimetics. Journal Progress in Materials Science, v. 54, p. 137-178, 2009.

MENGES, Achim. Material Computation - Introduction. Revista Architectural Design, v.82, n.2, p. 14-21, Março, 2012.

OLIVEIRA, A. L. Ensaio crítico sobre a primeira e a segunda antinomias de Kant. Síntese, Belo Horizonte, v. 28, n. 91, 2001.

OLIVEIRA, A. L. G. Cibernética, sistêmica e auto-organização para a composição de paisagens sonoras emergentes. 2011. Monografia (Disciplina de Introdução a Sistemas Inteligentes do Doutorado em Arte e Tecnologia) - Universidade de Brasilía.

OXMAN, Neri. Variable property rapid prototyping. Virtual and Physical Prototyping. v.6, n.1, p. 3-31. 2011.

PRIGOGINE, Ilya. O fim das certezas. São Paulo: Editora Unesp, 1996.

RAMOS, Joaquim Manuel Castelo. Auto-organização em sistemas de sinais. 2006. Dissertação (Mestrado em Design) - Universidade Técnica de Lisboa.

RUBIM, Renata. Desenhando a superfície. São Paulo: Editora Rosari, 2010.

RÜTHSCHILLING, Evelise Anicet. Design de superfície. Porto Alegre: Ed. da UFRGS, 2008.

TENU, Vlad. Minimal Surfaces as self-organizing systems. 2009. Dissertação (Master of Science in Adaptative Architecture and Computation) - University College London, Londres.

THEODOROU, Lida. Self-organizing Reaction-Diffusion Systems. 2012. Dissertação (Master of Science in Adaptive Architecture and Computation) - University College of London.

TURING, Alan. The Chemical Basis of Morphogenesis. Philosophical Transactions of the Royal Society (part B), v. 237, pp. 37-72, 1953. 